\title{
Associação entre Infecção por Heicobacter Pylori e Hipertensão Arterial Sistêmica: Metanálise
}

\author{
Association between Helicobacter Pylori Infection and Systemic Arterial Hypertension: A Meta-Analysis

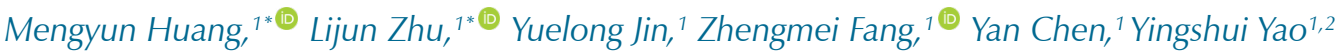 \\ Department of Epidemiology and Biostatistics, School of Public Health, Wannan Medical College/ Institute of Chronic Disease Prevention and \\ Control, ${ }^{1}$ Wuhu - China \\ Department of Medicine, Anhui College of Traditional Chinese Medicine, ${ }^{2}$ Wuhu - China \\ * Os autores Mengyun Huang e Lijun Zhu contribuíram igualmente para o manuscrito.
}

\section{Resumo}

Fundamento: Estudos epidemiológicos recentes demonstraram que alterações na microbiota e seus metabólitos estão associadas à hipertensão arterial sistêmica. A Helicobacter pylori (H. pylori) é um dos patógenos bacterianos mais comuns, e a possível associação entre a infecção por H. pylori e a hipertensão é controversa.

Objetivo: Este estudo teve o objetivo de esclarecer a associação entre eles e proporcionar uma nova base teórica para detectar a patogênese da hipertensão.

Métodos: Foram selecionados estudos caso-controle e transversais sobre a associação entre H. pylori e hipertensão, publicados de 1996 a 2019 indexados nos bancos de dados PubMed, Google Scholar, Chinese Wan Fang Data, e Chinese National Knowledge Infrastructure (CNKI). As razões de chance (RC) combinadas e o intervalo de confiança (IC) $95 \%$ foram estimados. O I I $^{2}$ foi realizado para avaliar a heterogeneidade estatística. O viés de publicação foi avaliado utilizando-se os testes de Beggs e de Egger. Os dados extraídos foram analisados no software Stata 12.0. A significância estatística foi definida com um p-valor $<0,05$.

Resultados: Foram cadastrados 17 estudos envolvendo 6376 casos de hipertensão e 10850 controles. A taxa de infecção por H. pylori em pacientes hipertensos e em controles foi de $64,9 \%$ e $56,3 \%$, respectivamente. Foi demonstrada uma associação significativamente positiva entre a infecção por $\mathrm{H}$. pylori e a hipertensão, com uma RC global de 2,07 (IC 95\%: 1,46-2,94; $p<0,05)$. A análise de subgrupos revelou que a prevalência de infecção por $H$. pylori foi associada à hipertensão na região da Ásia e no grupo de caso-controle, as RC (IC 95\%) foram 2,26 (1,51-3,38) e 2,53 (1,72-3,72), respectivamente. Depois de estratificar por métodos de detecção, ainda existiam diferenças entre os subgrupos (todos $\mathbf{p}<0,05)$.

Conclusão: Esta metanálise indicou que a infecção por H. pylori está associada positivamente à hipertensão.

Palavras-chave: Hipertensão; Pressão Arterial; Microbiota; Infecção; Epidemiologia, Helicobacter pylori; Fatores de Risco; Endotélio Vascular; Doenças Cardiovasculares; Metanálise.

\footnotetext{
Abstract

Background: Recent epidemiological studies have shown that alterations in microbiota and its metabolites are associated with systemic arterial hypertension. Helicobacter pylori (H. pylori) is one of the most common bacterial pathogens, and the potential association between H. pylori infection and hypertension are controversial.
}

Objective: This study aimed to clarify their association and provide a new theoretical basis for uncovering the pathogenesis of hypertension.

Methods: Case-control and cross-sectional studies on the association between H. pylori and hypertension published from 1996 to 2019 indexed in PubMed, Google Scholar, Chinese Wan Fang Data, and Chinese National Knowledge Infrastructure (CNKI). The pooled odds ratios $(\mathrm{OR})$ and $95 \%$ confidence interval $(\mathrm{Cl})$ were estimated. 12 was performed to evaluate the statistical heterogeneity. Publication bias was evaluated using Begg's and Egger's test. The extracted data was analyzed in Stata 12.0. Statistical significance was defined as p-value $<0.05$.

Results: A total of 17 studies involving 6,376 cases of hypertension and 10,850 controls were enrolled. H. pylori infection rate in hypertension patients and controls were $64.9 \%$ and $56.3 \%$, respectively. A significantly positive association was shown between $H$. pylori infection and hypertension with an overall OR of 2.07 (95\% Cl: 1.46-2.94; $p<0.05)$. Subgroup analysis revealed that the prevalence of H. pylori infection was associated with hypertension in the region of Asia and the case-control group, ORs (95\% CI) were 2.26 (1.51-3.38) and 2.53 (1.72-3.72), respectively. After stratifying by detection methods, differences still existed in subgroups (all $p<0.05$ ).

Correspondência: Yingshui Yao •

№ 22, Wenchang Road, Wuhu 241002, Anhui, China / № 18, Wuxia Shanxi Road, Wuhu. 241002, Anhui, China.

E-mail: yingshuiyao@163.com

Artigo recebido em 07/03/2020, revisado em 13/09/2020, aceito em 04/11/2020

DOI: https://doi.org/10.36660/abc.20200186 
Keywords: Hypertension; Blood Pressure; Microbiota; Infection; Epidemiology; Helicobacter Pylori; Risk Factors; Endothelium Vascular; Cardiovascular Diseases; Meta-Analysis.

Full texts in English - http://www.arquivosonline.com.br

\section{Introdução}

A hipertensão arterial sistêmica, também conhecida como pressão arterial alta ou elevada, é uma condição em que os vasos sanguíneos aumentam a pressão constantemente. De acordo com a Organização Mundial de Saúde (OMS), aproximadamente 1,13 bilhões de pessoa em todo o mundo têm hipertensão, e dois-terços delas vivem em países com renda baixa ou média. ${ }^{1} \mathrm{Na}$ China, cerca de 270 milhões de pessoas têm hipertensão, e sua prevalência é mais alta no Norte e mais baixa no Sul. ${ }^{2}$ Como um problema de saúde pública global, a hipertensão contribui para a carga de doenças cardíacas, acidente vascular cerebral, insuficiência renal, e outras doenças. ${ }^{3}$ Ela é considerada um distúrbio causal complexo, por ser influenciada pela interação entre vários fatores, tais como má alimentação, uso indevido de álcool, sedentarismo, tabagismo e fatores genéticos. ${ }^{4}$ Recentemente, estudos em seres humanos e animais demonstraram que alterações na microbiota e seus metabólitos estão associados à hipertensão arterial sistêmica. ${ }^{5,6}$

A H. pylori é um dos patógenos bacterianos mais comuns, e existe no piloro do estômago humano. ${ }^{7}$ A prevalência da $\mathrm{H}$. pylori, em países específicos varia de 18,9\% na Suíça a $87,7 \%$ na Nigéria. ${ }^{8}$ Ela já infectou mais da metade da população mundial. ${ }^{9}$ A infecção por $\mathrm{H}$. pylori causa inflamação crônica ativa com um recrutamento contínuo de neutrófilos para a mucosa gástrica inflamada. ${ }^{10}$ Além disso, outro estudo demonstrou que a microbiota intestinal facilita a disfunção vascular induzida por Angll e a hipertensão, causando a infiltração e a inflamação de células imunes vasculares. ${ }^{11} \mathrm{Um}$ estudo recente relatou que a soropositividade para $\mathrm{H}$. pylori está intimamente relacionada a aterosclerose, e a infecção por H. pylori pode contribuir para o desenvolvimento de doenças cardiovasculares. ${ }^{12}$ A pesquisa em animais demonstrou que a coinfecção de Chlamydia pneumoniae e H. pylori levou à disfunção endotelial vascular e aumentou a expressão de VCAM-1 em ratos. ${ }^{13}$ Esses achados destacam o papel importante desempenhado pela $\mathrm{H}$. pylori na regulação da disfunção endotelial e do sistema AngIII, e a possibilidade de a H. pylori estar envolvida no desenvolvimento da hipertensão.

Um estudo transversal envolvendo 5.246 participantes detectou uma associação positiva entre a infecção por $\mathrm{H}$. pylori e a hipertensão após o ajuste de possíveis fatores de confusão. ${ }^{14}$ Inversamente, o status de $\mathrm{H}$. pylori não foi significativamente diferente em pacientes com graus de hipertensão diferentes. ${ }^{15}$ Considerando a introdução acima e a diversidade de resultados sobre a infecção por $\mathrm{H}$. pylori e hipertensão, a necessidade de ser realizar um estudo para determinar a relação entre infecção por $\mathrm{H}$. pylori e hipertensão fica muito clara. Portanto, para investigar em mais detalhes o possível papel da infecção por $\mathrm{H}$. pylori na hipertensão, conduzimos uma metanálise para proporcionar uma base para a intervenção na hipertensão.

\section{Métodos}

\section{Coleta da literatura}

Todos os trabalhos sobre a relação entre H. pylori e hipertensão publicados entre 1996 e 2019 foram selecionados para esta metanálise. Foram pesquisados artigos cujos títulos e/ou resumos continham as expressões "H. pylori" ou "Helicobacter pylori" e "hipertensão" ou "pressão arterial alta", publicados em chinês nos bancos de dados Chinese Wanfang Data Knowledge Service Platform e Chinese National Knowledge Infrastructure (CNKI), e em inglês nos bancos de dados PubMed e Google Scholar. Por último, as referências foram filtradas novamente para evitar omissão, no processo de leitura de triagem dos artigos.

\section{Critérios de inclusão e de exclusão}

Todos os estudos que foram identificados pela pesquisa de literatura foram selecionados de acordo com os seguintes elementos essenciais: pacientes (indivíduos que foram diagnosticados de acordo com o padrão de diagnóstico de hipertensão arterial sistêmica); exposição (infecção por H. pylori); comparador (normotensão); resultado (associação entre infecção por $\mathrm{H}$. pylori e hipertensão); e desenho do estudo (estudo transversal ou caso-controle). Foram excluídos estudos ecológicos e de coorte, os que não agruparam por hipertensão e normotensão, aqueles em que o número de pacientes com $\mathrm{H}$. pylori em cada grupo não possa ser determinado, ou devido a possíveis erros. Nos casos em que o mesmo estudo apareceu em bancos de dados diferentes ou em que a população do estudo coincidiu, apenas o maior foi selecionado.

\section{Extração de dados e avaliação de qualidade no processo}

Em conformidade com o objetivo deste estudo, dois pesquisadores independentes selecionaram títulos e/ou resumos para serem incluídos nos artigos, e chegou-se a um consenso final pela avaliação de um terceiro especialista. Os estudos necessários foram lidos na íntegra, e as seguintes informações e características foram registradas: nome do primeiro autor, ano de publicação, país, tipo de estudo, média de idade, número de participantes, e testes de infecção por H. pylori.

\section{Análise estatística}

Todas as análises estatísticas foram realizadas utilizando-se o software Stata 12.0. As razões de chance $(\mathrm{RC})$ combinadas, com os intervalos de confiança (IC) de 95\% correspondentes, foram consideradas tamanho de efeito para todos os estudos qualificados. Dois métodos (o teste de Cochran e a estatística $\left.I^{2}\right)$ firam usados para avaliar a heterogeneidade estatística 
entre os dados resumidos: o $p<0,05$ foi considerado estatisticamente significativos quanto à heterogeneidade, e a estatística I ${ }^{2}$ sugere heterogeneidade significativa com um valor $>50 \% .{ }^{16} \mathrm{Um}$ modelo de efeito randômico foi usado para calcular a estimativa de tamanho de efeito geral para esta metanálise. Para examinar as fontes de heterogeneidade entre os estudos qualificados, foram realizadas análises de subgrupos de acordo com características diferentes, tais como desenho do estudo (estudos transversais ou caso-controle), país do estudo (asiáticos ou ocidentais), e testes para infecção por H. pylori (teste respiratório com ureia marcada, testes sorológicos ou outros). Os testes respiratórios com ureia marcada (UBT) incluíram o 13C-UBT e o 14C-UBT, os testes sorológicos incluíram o método Colloidal gold e o ELISA. Outros testes incluíram a Coloração de Giemsa e os casos em que as informações não estavam disponíveis (N/D). A análise de sensibilidade foi realizada para avaliar os efeitos do estudo específico na estimativa de efeitos resumidos e na estabilidade dos resultados. Os testes de regressão de Begg e Egger foram usados para avaliar o viés de publicação. Foi realizado o método de metarregressão para dados de medição, tais como, tamanho da amostra, média de idade e razão de sexos. Um p valor bicaudal $<0,05$ foi definido como estatisticamente significativo.

\section{Resultados}

\section{Características básicas dos artigos ${ }^{17-33}$}

Foi apresentado um fluxograma de estudos para revisão sistemática na Figura 1. Desses estudos, foram excluídos 80 estudos duplicados, e, em seguida, com base nos títulos e resumos dos artigos restantes, foram excluídos 57 estudos. Ao final, 17 publicações foram selecionadas após realizar o exame do texto dessas 22 publicações na íntegra, conforme especificado no fluxograma.

No total, 6 estudos transversais e 11 estudos caso-controle foram selecionados na metanálise, no período de 1996 a 2019. Entre os 17.226 participantes, a prevalência de H. pylori em 6.376 pacientes hipertensos foi de 64,9 , e em 10.850 normotensos foi de $56,3 \%$. As principais características dos estudos incluídos nesta revisão foram apresentadas na Tabela $1 .{ }^{17-33}$

\section{Resultados da metanálise}

Com base no modelo de efeitos randômicos da Figura 2, a estimativa geral das RC combinadas (IC 95\%) de $\mathrm{H}$. pylori e hipertensão foi 2,07 $(1,46-2,94)$, o que era estatisticamente significativo $(p<0,001)$.

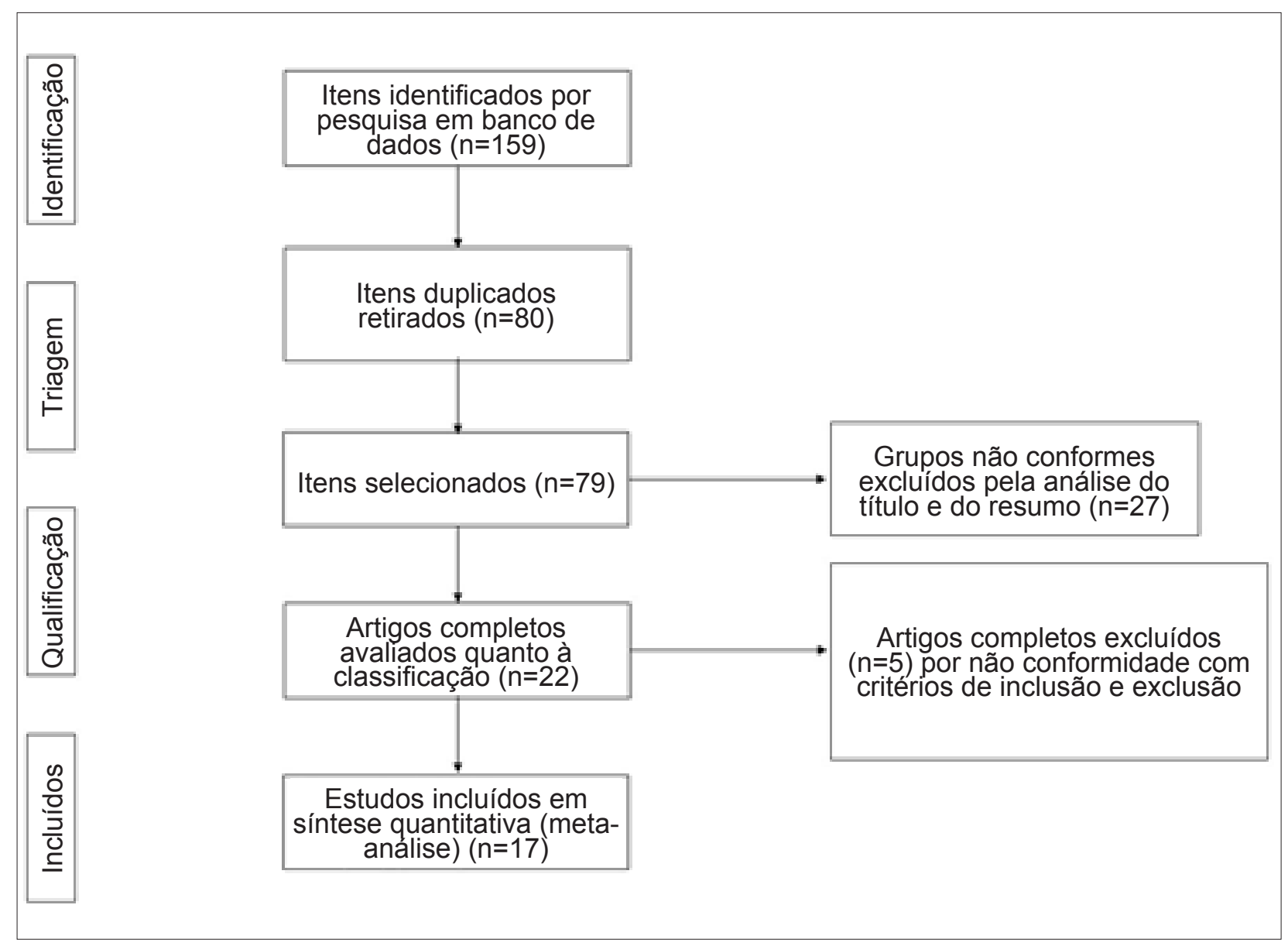

Figura 1 - Fluxograma dos estudos incluídos na meta-análise. 


\begin{tabular}{|c|c|c|c|c|c|c|c|c|c|}
\hline Estudos & Ano & País & $\begin{array}{c}\text { Constatação da } \\
\text { H. pylori }\end{array}$ & $\begin{array}{l}\text { Hipertensão } \\
\text { (H. pylori + / -) }\end{array}$ & $\begin{array}{l}\text { Normotensos } \\
\text { (H. pylori }+/- \text { ) }\end{array}$ & $\begin{array}{c}\text { Média } \\
\text { de idade }\end{array}$ & Desenho do estudo & $\begin{array}{l}\text { Razão de } \\
\text { sexos }\end{array}$ & $\underset{p}{\operatorname{limiar}}$ \\
\hline Yang ${ }^{17}$ & 2016 & China $^{\#}$ & 14C-UBT & $78(66 / 12)$ & $78(37 / 41)$ & $\mathrm{N} / \mathrm{D}$ & Estudo caso-controle & 0,79 & 0,05 \\
\hline $\operatorname{Liu}^{18}$ & 2015 & China $^{\#}$ & 14C-UBT & $150(125 / 25)$ & $150(71 / 79)$ & 63,60 & Estudo caso-controle & 1,04 & 0,05 \\
\hline Huai $^{19}$ & 2014 & China $^{\#}$ & 13C-UBT & $535(338 / 197)$ & $1051(232 / 819)$ & 68,30 & Estudo transversal & 2,68 & 0,05 \\
\hline $\mathrm{Ma}^{20}$ & 2015 & China $^{\#}$ & $\begin{array}{l}\text { Coloração de } \\
\text { Giemsa }\end{array}$ & $112(42 / 70)$ & $170(53 / 117)$ & $N / D$ & Estudo caso-controle & $\mathrm{N} / \mathrm{D}$ & 0,05 \\
\hline $\mathrm{Chu}^{21}$ & 2014 & China $^{\#}$ & IgG Colloidal gold & $150(67 / 83)$ & $50(14 / 36)$ & 60,85 & Estudo caso-controle & 1,41 & 0,05 \\
\hline $\mathrm{Hu}^{22}$ & 2017 & China $^{\#}$ & N/D & $176(82 / 94)$ & $568(224 / 344)$ & 52,53 & Estudo transversal & 1,23 & 0,05 \\
\hline $\operatorname{Lip}^{23}$ & 1996 & $\begin{array}{l}\text { Reino } \\
\text { Unido } \$\end{array}$ & $\begin{array}{c}E L I S A \geq 8 \\
\text { unidades/ml). }\end{array}$ & $124(106 / 18)$ & $38(25 / 13)$ & 53,06 & Estudo caso-controle & 1,03 & 0,05 \\
\hline $\operatorname{Liu}^{24}$ & 2007 & China $^{\#}$ & ELISA & 488(189/299) & $942(317 / 625)$ & 46,56 & Estudo transversal & 0,71 & 0,05 \\
\hline $\operatorname{Sun}^{25}$ & 2018 & China $^{\#}$ & 14C-UBT & $90(76 / 14)$ & $65(34 / 31)$ & 44,74 & Estudo caso-controle & 1,12 & 0,05 \\
\hline $\mathrm{Li}^{26}$ & 1999 & China $^{\#}$ & $\lg$ ELISA & $42(16 / 26)$ & $60(18 / 42)$ & 55,41 & Estudo caso-controle & 1,62 & 0,05 \\
\hline $\mathrm{Zhao}^{27}$ & 2019 & China $^{\#}$ & 14C-UBT & $102(43 / 59)$ & $102(18 / 84)$ & 47,85 & Estudo caso-controle & 0,94 & 0,05 \\
\hline Migneco ${ }^{28}$ & 2003 & Itália $^{\$}$ & 13C-UBT & $72(40 / 32)$ & $70(35 / 35)$ & 52,51 & Estudo caso-controle & 0,95 & 0,05 \\
\hline Vahdat $^{29}$ & 2013 & Irã ${ }^{\#}$ & ELISA $>30 \mathrm{RU} / \mathrm{ml}$ & $459(316 / 143)$ & $1295(764 / 531)$ & 40,79 & Estudo transversal & 0,97 & 0,05 \\
\hline Zheng $^{30}$ & 2014 & China $^{\#}$ & $\begin{array}{c}\text { Coloração de } \\
\text { Giemsa }\end{array}$ & $112(42 / 70)$ & $170(53 / 117)$ & 52,19 & Estudo caso-controle & 1,29 & 0,05 \\
\hline Sung ${ }^{31}$ & 2003 & Coreia $^{\#}$ & $\mathrm{ELISA} \geq 6 \mathrm{U} / \mathrm{mL}$ & $2838(1964 / 874)$ & $5509(3818 / 1691)$ & 48,06 & Estudo transversal & 1,36 & 0,05 \\
\hline Sotuneh $^{32}$ & 2014 & Itália ${ }^{s}$ & ELISA >20 ur/ml & $808(606 / 202)$ & 492(385/107) & 69,23 & Estudo transversal & $\mathrm{N} / \mathrm{D}$ & 0,05 \\
\hline S.VSM ${ }^{33}$ & 2012 & Índia ${ }^{\#}$ & $\begin{array}{c}\text { IgG ELISA > } \\
40 \mathrm{ur} / \mathrm{ml}\end{array}$ & $40(18 / 22)$ & $40(9 / 31)$ & 44,09 & Estudo caso-controle & 1,05 & 0,05 \\
\hline
\end{tabular}

UBT: teste respiratório com ureia marcada; ELISA: ensaio de imunoabsorção enzimática; N/D: não disponível; * Razão de sexos: masculino/feminino; \# países asiáticos; \$ paises ocidentais.

Análise de subgrupos por região, desenho de estudo, e método de teste

Para a análise por subgrupo por região (em países asiáticos, 5372 pacientes hipertensos e 10250 normotensos, e em países ocidentais, 1004 pacientes hipertensos e 600 normotensos), a infecção por $\mathrm{H}$. pylori foi associada ao risco de hipertensão em países asiáticos (RC 2,26, IC 95\% $\left.1,51-3,38 ; I^{2}=95,1 \%, p<0,05\right)$, e não houve diferenças significativas nos países ocidentais.

$\mathrm{Na}$ análise por subgrupo por desenho do estudo, a RC combinada para infecção por $\mathrm{H}$. pylori e hipertensão foi de 2,53 (IC 95\% 1,72-3,72; I I = 72,7\%, p < 0,05) nos estudos caso-controle. Não se observaram diferenças estatísticas nos estudos transversais (Figuras 3 e 4).

$\mathrm{Na}$ análise por subgrupo que utilizou o UBT para testar quanto à infecção por $\mathrm{H}$. pylori, esta foi associada a um risco mais alto de hipertensão (RC 4,13, IC 95\% 2,60-6,54; $\left.\mathrm{I}^{2}=76,7 \%, \mathrm{p}<0,05\right)$. A infecção por $\mathrm{H}$. pylori também foi associada a um risco mais alto de hipertensão na análise por subgrupo que utilizou testes sorológicos (RC 1,33, IC 95\% $\left.1,04-1,68 ; I^{2}=77,1 \%, p<0,05\right)$ (Figura 5).

\section{Viés de publicação}

O teste de Begg foi utilizado para avaliar o viés de publicação com o gráfico de funil (Figura 6). Houve uma assimetria no gráfico de funil para os estudos selecionados. $O$ viés de publicação significativo também foi identificado no teste de regressão de Egger ( $p=0,047)$.

\section{Análise de sensibilidade}

Realizamos uma análise de sensibilidade para testar as fontes de heterogeneidade e avaliamos a estabilidade dos resultados. Os resultados da análise de sensibilidade demonstraram que nenhum estudo específico teve influência extrema na razão de chances combinada (Figura 7).

\section{Metarregressão}

Estudos específicos sem média de dados $(\mathrm{n}=2)$ ou sem dados de sexo masculino ou feminino $(n=2)$ foram excluídos. Os resultados de metarregressões univariadas mostram a ausência de efeitos significativos de tamanho de amostra ( $p$ $=0,181)$, média de idade $(p=0,542)$, razão de sexos $(p=$ 0,367) na associação entre a infecção por H. pylori e o risco de hipertensão nos estudos (Material Suplementar: Figuras suplementares 1-3).

\section{Discussão}

Até onde sabemos, este é o primeiro estudo de revisão sistemática que demonstra a relação entre a infecção por H. pylori e a hipertensão. Os achados demonstraram 


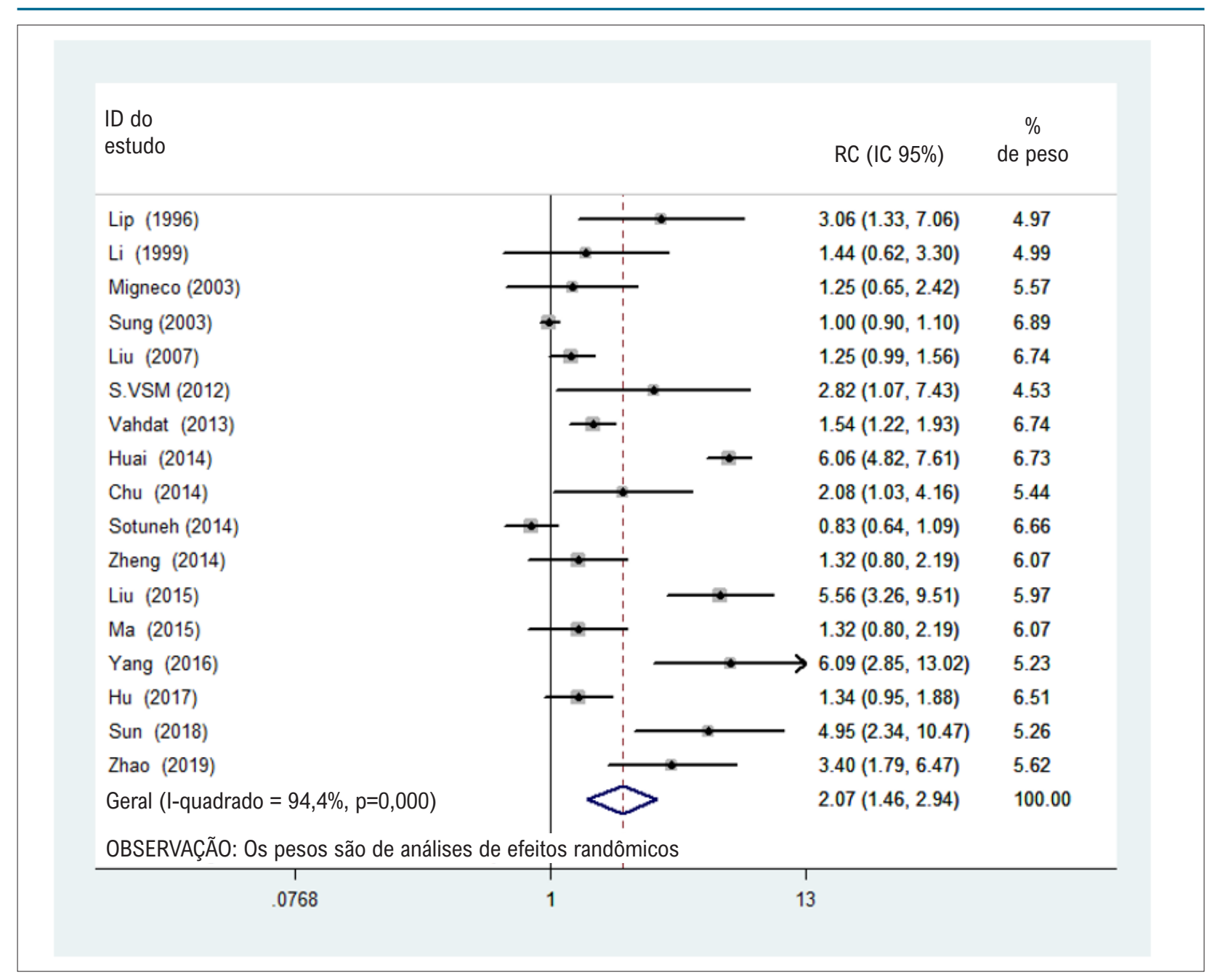

Figura 2 - Gráfico de floresta da associação entre infecção por H. pylori infecção e hipertensão.

que a prevalência da infecção por $\mathrm{H}$. pylori foi associada positivamente à hipertensão, e esse resultado foi consistente no grupo de caso-controle na análise por subgrupo. Mendel et al. ${ }^{34}$ foram os primeiros a levantar a hipótese da relação entre a infecção por H. pylori e as doenças cardíacas coronárias. Depois disso, alguns acadêmicos realizaram pesquisas sobre a infecção por $\mathrm{H}$. pylori em doenças cardiovasculares. ${ }^{35}$

Um estudo de coorte prévio demonstrou que indivíduos infectados por $\mathrm{H}$. pylori, com diagnóstico positivo de gastrite ativa crônica, tinham o risco $29 \%$ mais alto de desenvolver hipertensão durante o período de acompanhamento. ${ }^{36}$ Um estudo chinês indicou que a infecção por $\mathrm{H}$. pylori foi independentemente associada a PAD mais alta, mas não PAS mais alta depois de se ajustar as covariáveis. ${ }^{14}$ Entretanto, vários estudos demonstraram que a infecção por $\mathrm{H}$. pylori não influenciou a pressão arterial. ${ }^{37,38}$ Obviamente, não podemos concluir que a infecção por $\mathrm{H}$. pylori facilita a hipertensão. Entretanto, nossa metanálise agora sustenta a associação entre a infecção por $\mathrm{H}$. pylori e a hipertensão.

A prevalência da infecção por H. pylori varia notadamente em vários países e regiões. As infecções por H. pylori em países asiáticos eram comuns e distribuídas por uma grande extensão. A taxa média de infecção na China foi de 58,07\%, com $50 \%$ na faixa etária entre 10 e 20 anos. ${ }^{39} \mathrm{~A}$ investigação epidemiológica demonstrou que a cepa positiva para o gene A associado a citocina (gene cagA) de várias áreas geográficas apresenta clara diferenciação filogeológica. Mais de 90\% dos isolados de $\mathrm{H}$. pylori de países da Ásia Ocidental, tais como a China e o Japão, contêm proteína cagA, enquanto apenas $60 \%$ a $70 \%$ dos isolados de $\mathrm{H}$. pylori em países ocidentais, tais como os Estados Unidos, contêm a proteína cagA. ${ }^{40}$ Além disso, Migneco A et al. ${ }^{28}$ identificaram que apenas entre os pacientes com resultado positivo alto para cagA a PAD diminuiu mais claramente depois de o $\mathrm{H}$. pylori ter sido erradicado, e isso pode estar ligado ao relacionamento molecular entre o antígeno cagA da H. pylori e alguns peptídeos expressos por células endoteliais e células de músculo liso. É notável que a RC da hipertensão em populações asiáticas com infecção por $\mathrm{H}$. pylori foi 2,26 vezes maiores do que as de sujeitos normotensos em nosso estudo. Esses resultados sugerem que a origem étnica pode ter um possível impacto na relação entre a infecção por H. pylori e a hipertensão. 


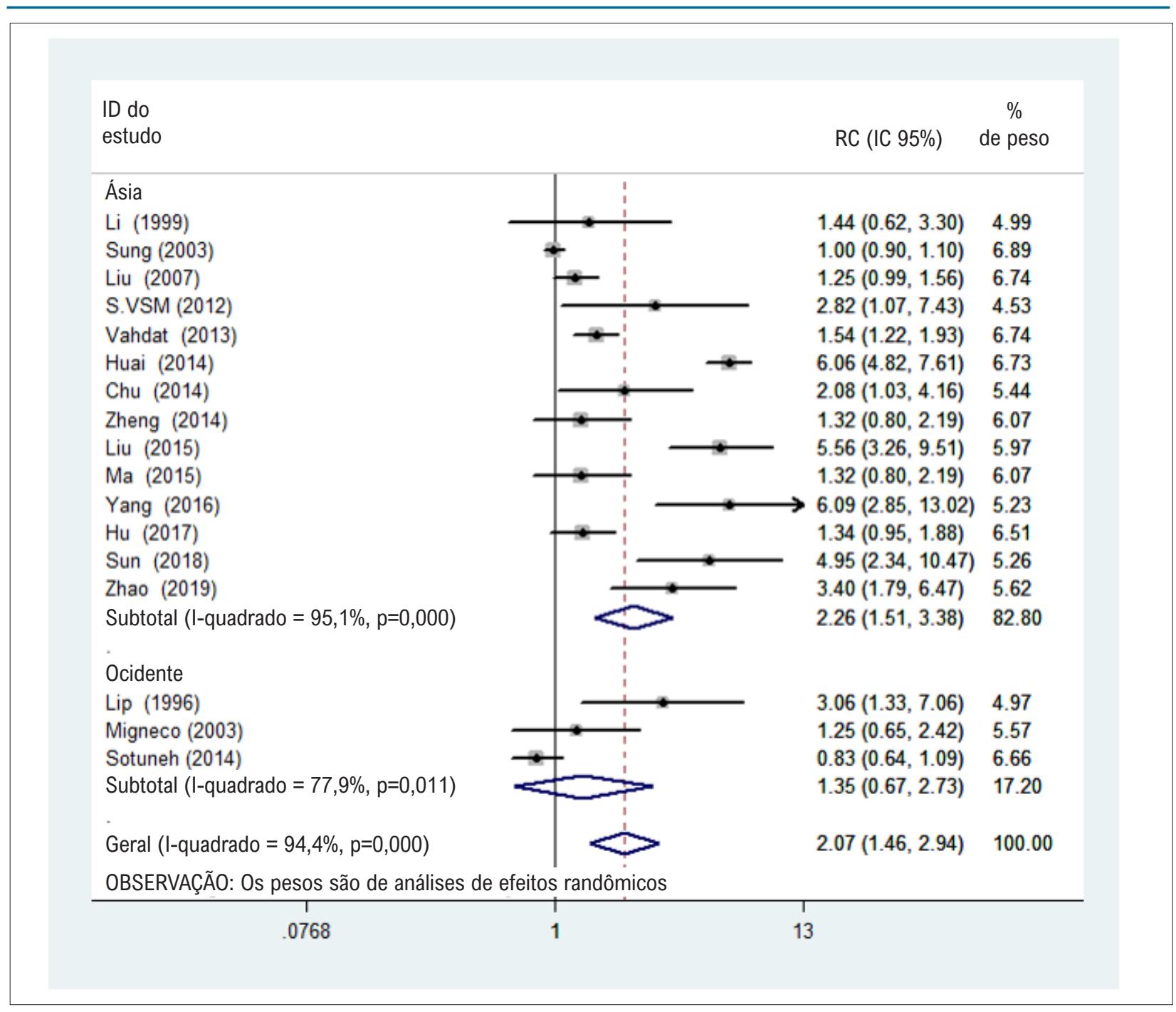

Figura 3 - Gráfico de floresta de análises de subgrupos comparando a Ásia ao Ocidente.

Outro problema para se determinar a infecção por H. pylori e hipertensão é o método utilizado para testar a infecção por H. pylori. Embora os testes sorológicos sejam uma forma de triagem comum, eles não garantem a identificação da infecção por H. pylori ativa. ${ }^{41}$ A precisão do diagnóstico utilizando-se o UBT para se detectar a infecção por H. pylori na população asiática, especialmente o 13C-UBT, que tinha uma precisão diagnóstica impressionante, com uma sensibilidade de $97 \%$ e uma especificidade de $96 \%{ }^{42}$ Em nossa análise por subgrupo, o risco de hipertensão era mais alto quando a infecção por $\mathrm{H}$. pylori foi determinada utilizando-se um UBT, em comparação com outros testes.

Os mecanismos que ligam a infecção por H. pylori à hipertensão ainda não estão claros. Há várias hipóteses que sustentam a relação, e uma das mais plausíveis é a dos níveis de citocinas inflamatórias. Epstein et al. ${ }^{43}$ indicaram que a inflamação crônica causada pela infecção por $\mathrm{H}$. pylori pode levar à aterosclerose avançada, consequentemente. Além disso, um estudo prospectivo demonstrou que a $\mathrm{H}$. pylori tinha uma associação positiva com LDL alto e HDL baixo. ${ }^{44}$ Os lipídeos, como parte integral da membrana celular, têm um papel importante no desenvolvimento da hipertensão. ${ }^{45}$ Especula-se que a infecção por $\mathrm{H}$. pylori leva ao metabolismo anormal de LDL-C, HDL-C e TC, o que, por sua vez, resulta em hipertensão. A infecção por H. pylori destrói a tolerância imune e causa reação autoimune, que pode fazer parte da patogênese da hipertensão. ${ }^{46,47}$ Identificou-se que a idade é um preditor independente da infecção por H. pylori, e há uma tendência crescente de prevalência com a idade, apesar de não existir diferença de prevalência entre os sexos. ${ }^{48}$ Além disso, os homens têm duas vezes mais probabilidade de desenvolver doenças cardiovasculares que as mulheres, pelo menos abaixo dos 60 anos. ${ }^{49}$ Entretanto, nosso estudo mostra a ausência de efeitos significativos de idade e sexo na associação entre a infecção por $\mathrm{H}$. pylori e o risco de hipertensão. É possível que diferenças étnicas, avaliação da infecção por $\mathrm{H}$. pylori, tamanho de amostras, e possíveis fatores de confusão contribuam para a existência de discrepâncias. 


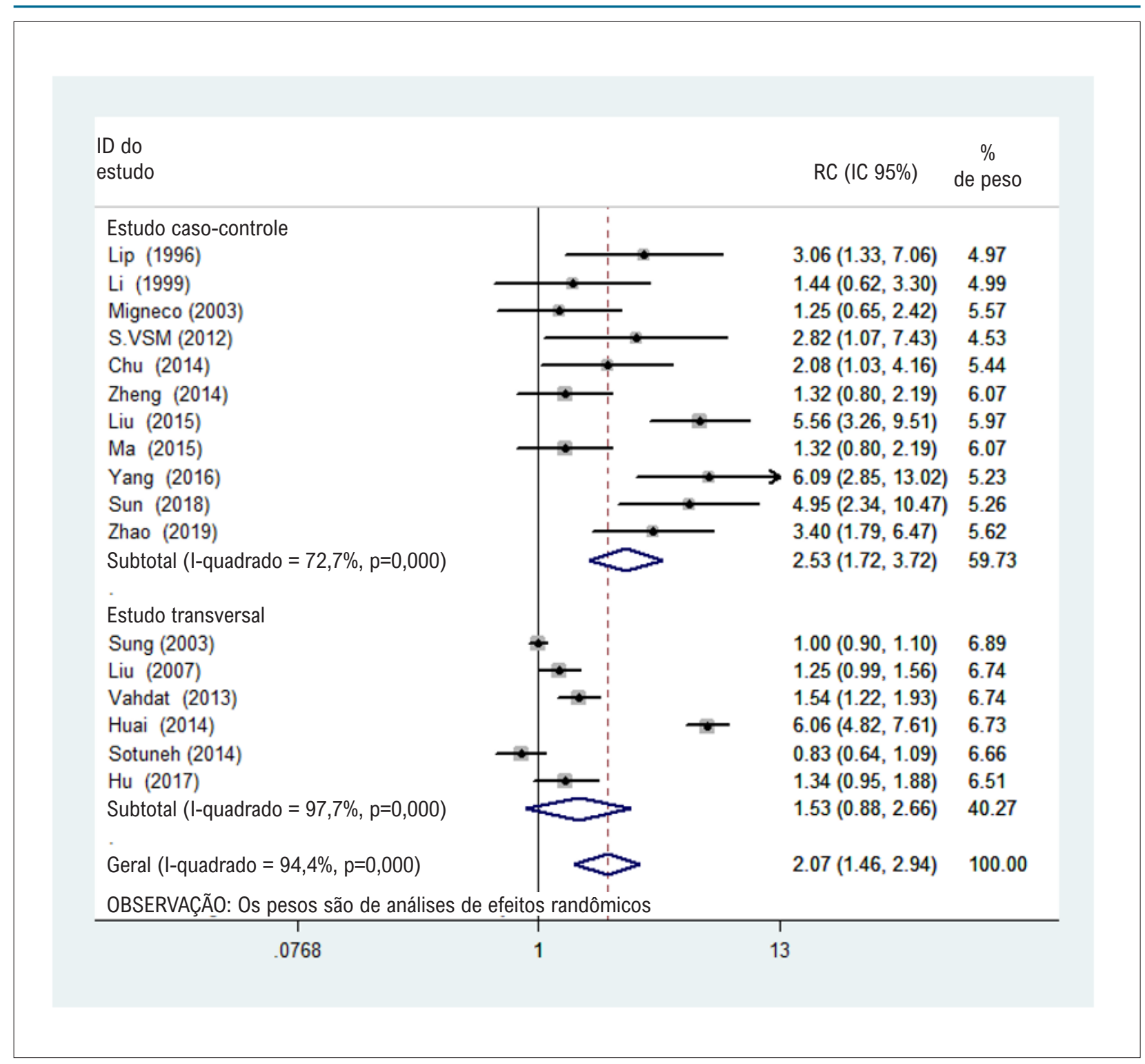

Figura 4 - Gráfico de floresta de análises de subgrupos estudos caso-controle a estudos transversais.

\section{Limitações}

Há várias limitações em nosso estudo. Primeiramente, todos os estudos incluídos foram estudos observacionais, o que dificultou a estimativa de uma associação causal. Em segundo lugar, foi identificada uma heterogeneidade nas metanálises e não foi possível ajustar variáveis com o potencial de serem fatores de confusão por outras informações não acessíveis. Em terceiro lugar, mais estudos originais foram realizados na população asiática, e houve um viés de publicação na metanálise.

\section{Conclusões}

Em conclusão, nossos resultados indicaram que a infecção por H. pylori está associada positivamente à hipertensão. As estratégias de prevenção da infecção por $\mathrm{H}$. pylori e a erradicação da H. pylori podem ter um efeito significativo na prevenção e no tratamento da hipertensão, e devem ser avaliadas em mais detalhes.

\section{Author Contributions}

Concepção e desenho da pesquisa: Huang M, Zhu L. Obtenção de dados: Jin Y, Fang Z. Análise e interpretação dos dados: Fang Z. Análise estatística: Jin Y. Obtenção de financiamento: Yao Y, Chen Y. Redação do manuscrito: Huang M, Zhu L. Revisão crítica do manuscrito quanto ao conteúdo intelectual importante: Yao Y, Chen Y.

\section{Potencial Conflito de Interesse}

Declaro não haver conflito de interesses pertinentes. 


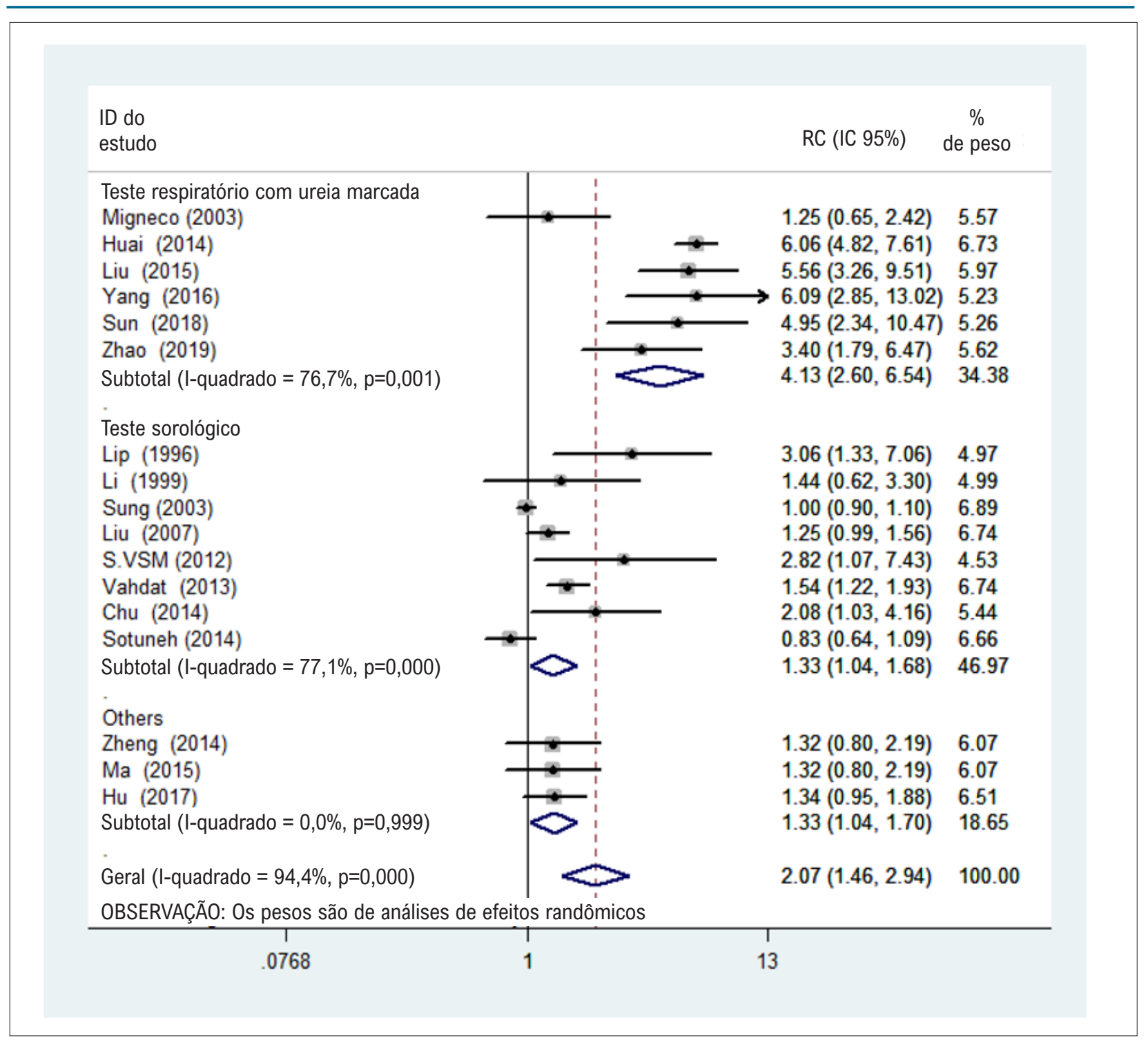

Figura 5 - Gráfico de floresta de análises de subgrupos comparando vários métodos de diagnóstico.

\section{Fontes de Financiamento}

O presente estudo foi financiado por National Natural Science Foundation of China (№ 81874280 and № 81673266); Anhui Provincial Natural Science Foundation (№ 1808085QH283 and № 1808085MH297); Key Research and Development Program of Anhui Province (№ 1804h08020261); Key Projects of Anhui Provincial Department of Education (№ KJ2019A0405).

\section{Vinculação Acadêmica}

Não há vinculação deste estudo a programas de pósgraduação.

\section{Aprovação Ética e Consentimento Informado}

Este artigo não contém estudos com humanos ou animais realizados por nenhum dos autores. 


\section{Artigo Original}

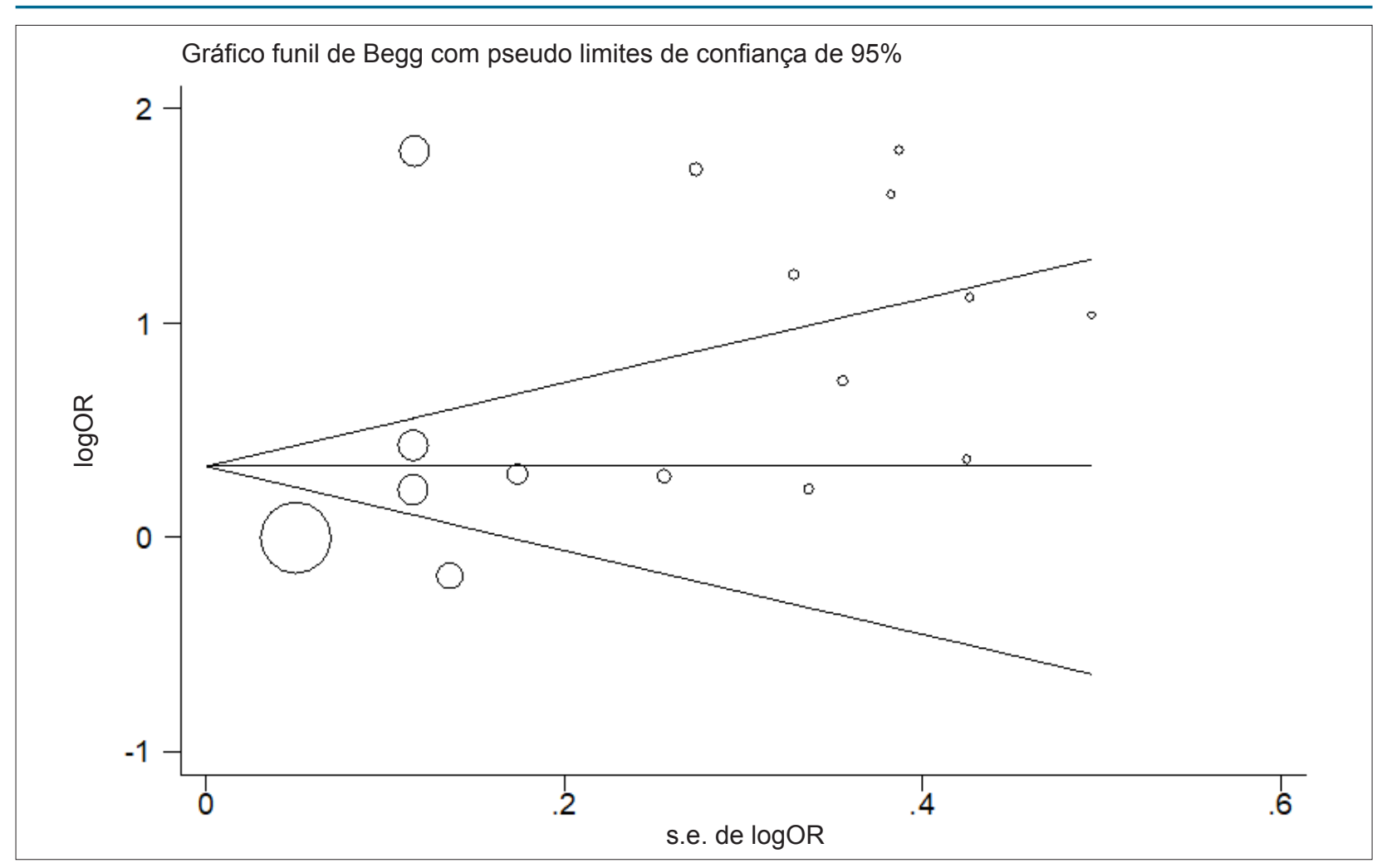

Figura 6 - Gráfico de funil com pseudolimite de confiança de 95\%.

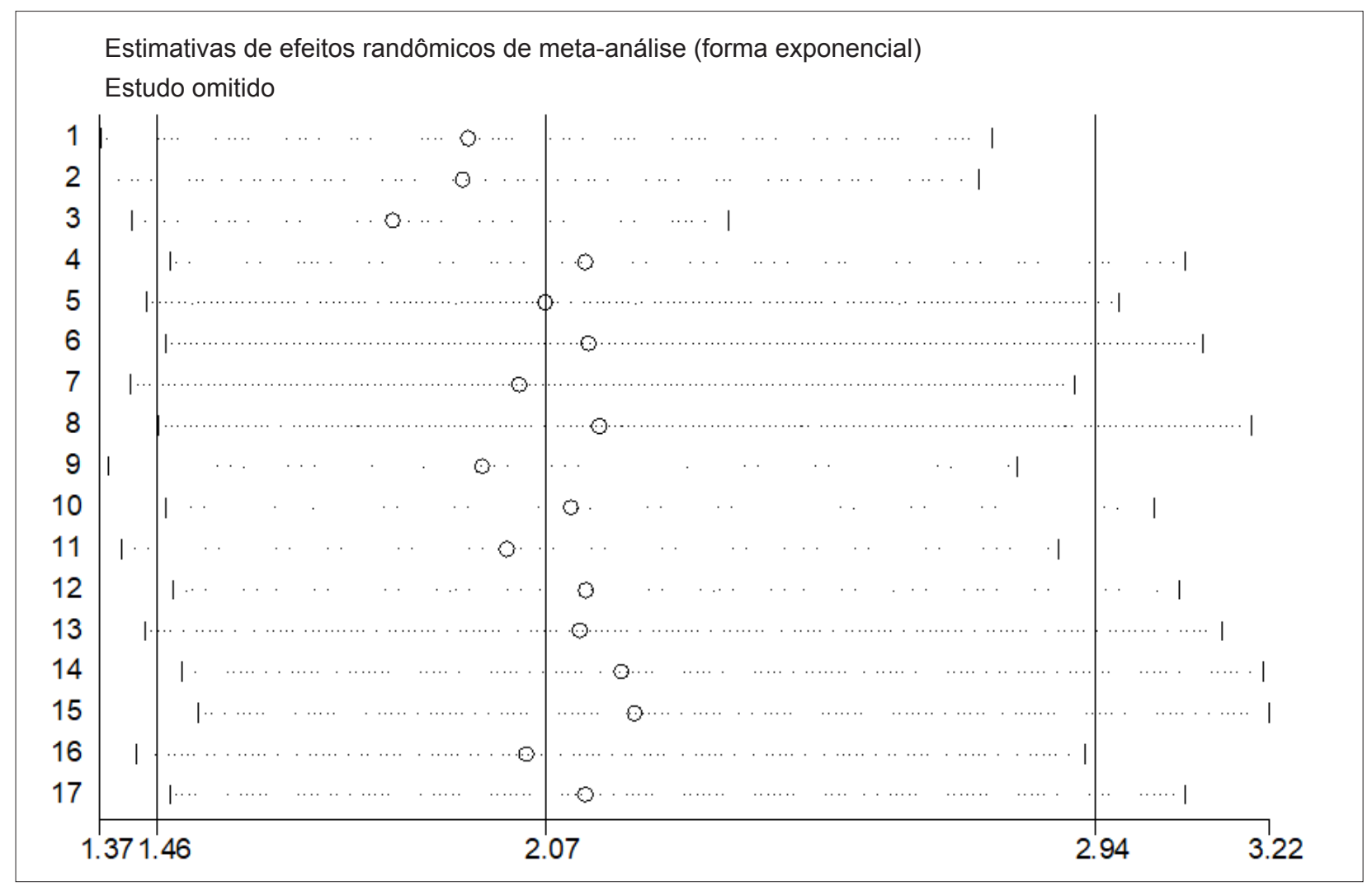

Figura 7 - Análise de sensibilidade para associação entre H. pylori e hipertensão. 


\section{Referências}

1. Subasinghe AK, Arabshahi S, Busingye D, Evans RG, Walker KZ, Riddell $M A$, et al. Association between salt and hypertension in rural and urban populations of low to middle income countries: A systematic review and meta-analysis of population based studies. Asia Pacific Clin Nutr. 2016;25(2):402-13.

2. Li Y, Wang L, Feng X, Zhang M, Huang Z, Deng Q, et al. Geographical variations in hypertension prevalence, awareness, treatment and control in china: Findings from a nationwide and provincially representative survey. J Hypertens. 2018;36(1):178-87

3. Messerli FH, Williams B, Ritz E. Essential hypertension. Lancet. 2007;370(9587):591-603.

4. Forman JP, Stampfer MJ, Curhan GC. Diet and lifestyle risk factors associated with incident hypertension in women. Jama. 2009;302(4):401-11.

5. Pevsner-Fischer M, Blacher E, Tatirovsky E, Ben-Dov IZ, Elinav E. The gut microbiome and hypertension. Curr Opin Nephrol Hypertens. 2017;26(1):1-8.

6. Yang T, Santisteban MM, Rodriguez V, Li E, Ahmari N, Carvajal JM, et al. Gut dysbiosis is linked to hypertension. Hypertension. 2015;65(6):1331-40.

7. Brown LM. Helicobacter pylori: Epidemiology and routes of transmission. Epidemiol Rev. 2000;22(2):283-97.

8. Hooi JKY, Lai WY, Ng WK, Suen MMY, Underwood FE, Tanyingoh D, et al. Global prevalence of helicobacter pylori infection: Systematic review and meta-analysis. Gastroenterology. 2017;153(2):420-9.

9. Leja M, Axon A, Brenner H. Epidemiology of helicobacter pylori infection. Helicobacter. 2016;21 (Suppl 1):3-7.

10. Innocenti M, Thoreson AC, Ferrero RL, Stromberg E, Bolin I, Eriksson L, et al. Helicobacter pylori-induced activation of human endothelial cells. Infect immun. 2002;70(8):4581-90.

11. Ma J, $\mathrm{Li} \mathrm{H}$. The role of gut microbiota in atherosclerosis and hypertension. Front Pharmacol. 2018;9:1082.

12. Choi JM, Lim SH, Han YM, Lee H, Seo JY, Park HE, et al. Association between helicobacter pylori infection and arterial stiffness: Results from a large crosssectional study. PloS One. 2019;14:e0221643.

13. Liuba P, Pesonen E, Paakkari I, Batra S, Andersen L, Forslid A, et al. Coinfection with chlamydia pneumoniae and helicobacter pylori results in vascular endothelial dysfunction and enhanced vcam-1 expression in apoeknockout mice. J Vasc Res. 2003;40(2):115-22.

14. Wan Z, Hu L, Hu M, Lei X, Huang Y, Lv Y. Helicobacter pylori infection and prevalence of high blood pressure among chinese adults. J Hum Hypertens. 2018;32(2):158-64.

15. Lu C, Jia H, Xu A, Tang J, Xu G, Yue W, et al. Helicobacter pylori infection and pepsinogen levels have clinical significance in hypertension patients. Int J Clin Exp Med. 2014;7(12):5675-80

16. Higgins JP, Thompson SG, Deeks JJ, Altman DG. Measuring inconsistency in meta-analyses. BMJ. 2003;327(7414):557-60.

17. Guang Y, Qian-sheng W, Yun-xia L, Xin-rong H, Dong-liang L. Research of helicobacter pylori infection and change of c-reactive protein in patients with essential hypertension. Chin J Nosocomiol.26:2735-7.

18. Fei-fei L, Jun S, Hai-yan G. Helicobacter pylori infection and its correlation in 150 patients with hypertension. Prev Treat Cardiovasc Dis. 2015:42-3.

19. Yong-jun H, Gui-chi Z, Hui-ling L. Relationship between helicobacter pylori infection and primary hypertension and hypertensive heart disease. Clin J Med Offic. 2014;42:1113-1114+1121

20. Yun-ma S, Xue-mei W, Mei-yue C. Clinical analysis of hypertension with peptic ulcer. Health Vocational Education. 2015;33:144-5.

21. Hong-jiu C, Wen-dong Y. Correlation between carotid artery atherosclerosis and helicobacter pylori infection in patients with essential hypertension. Pract J Card Cerebr Pneumol Vasc Dis. 2014;22:22-4.
22. Ming-xi H, Li Y, Yuan-yuan W, Jin-ying G, Lan L. Assess of blood vessel condition on different levels of blood pressure and analysis of the relevant risk factors. Chin J Laborat Diagn. 2017;21:1221-5.

23. Lip GH, Wise R, Beevers G. Association of helicobacter pylori infection with coronary heart disease. Study shows association between $\mathrm{h}$ pylori infection and hypertension. BMJ. 1996;312:250-1.

24. Liu L, Liu Y, Tong W, Ye H, Zhang X, Cao W, et al. Pathogen burden in essential hypertension. Circulation. 2007;71:1761-4

25. Ping S, Jia-he S, Qing-xia X, Li-li Z, Xi-tian H. Serum levels of tnf- $\mathrm{C}$, il-6 and il-8 inhypertensive patients with different types of helicobacter pylori infection. Infect Dis Info. 2018;31:165-7.

26. Dai-yu L, Hua Z, Liang-hai C, Qing W. A preliminary study on the relationship between serum helicobacter pylori antibody detection and coronary heart disease. J South Med Univ. 1999:200-1.

27. Wei-hua Z. Correlation analysis between helicobacter pylori infection and lipid metabolism in patients with essential hypertension. Mod DiagnTreat. 2019;30:2292-3.

28. Migneco A, Ojetti V, Specchia L, Franceschi F, Candelli M, Mettimano M et al. Eradication of helicobacter pylori infection improves blood pressure values in patients affected by hypertension. Helicobacter. 2003;8:585-9.

29. Vahdat K, Pourbehi MR, Ostovar A, Hadavand F, Bolkheir A, Assadi M, et al Association of pathogen burden and hypertension: The persian gulf healthy heart study. Am J Hypertens. 2013;26:1140-7.

30. Zheng-fa Z. Clinical analysis of peptic ulcer in patients with hypertension China Health Care Nutrition. 2014:72-3.

31. Sung KC, Suh JY, Kim BS, Kang JH, Kim H, Lee MH, et al. High sensitivity C-reactive protein as an independent risk factor for essential hypertension. Am J Hypertens. 2003;16:429-33.

32. Sotuneh N, Hosseini SR, Shokri-Shirvani J, Bijani A, Ghadimi R. Helicobacte pylori infection and metabolic parameters: Is there an association in elderly population? Int J Prev Med. 2014; 5:1537-42.

33. S. VSM, Kutty AVM, Annamalai N. Helicobacter pylori infection and hypertension: Is there an association? Biomed Res.2012 (0970-938X)

34. Mendall MA, Goggin PM, Molineaux N, Levy J, Toosy T, Strachan D, et al. Relation of helicobacter pylori infection and coronary heart disease. Br Heart J. 1994;71:437-9

35. Kim YI, Kim YA, Lee JW, Kim HJ, Kim SH, Kim SG, et al. Effect of $<\mathrm{i}>$ helicobacter pylori $</ \mathrm{i}>$ treatment on long-term mortality in patients with hypertension. Gut Liver. 2020;14:47-56.

36. Barnes RJ, Uff JS, Dent JC, Gear MW, Wilkinson SP. Long-term follow up of patients with gastritis associated with helicobacter pylori infection. BritJ Gen Pract. 1991;41:286-8.

37. Kopacova M, Koupil I, Seifert B, Fendrichova MS, Spirkova J, Vorisek V, et al. Blood pressure and stature in helicobacter pylori positive and negative persons. World J Gatroenterol.2014;20:5625-31.

38. Gunji T, Matsuhashi N, Sato H, Fujibayashi K, Okumura M, Sasabe N, et al. Helicobacter pylori infection is significantly associated with metabolic syndrome in the japanese population. Am J Gastroenterol. 2008;103:3005-10.

39. Wang KJ, Wang RT. Meta-analysis on the epidemiology of helicobacter pylori infection in china. Zhonghua liu xing bing xue za zhi $=$ Zhonghua liuxingbingxue zazhi. 2003;24:443-6.

40. Yamaoka Y. Helicobacter pylori typing as a tool for tracking human migration Clin Microbiol.Infect. 2009;15:829-34.

41. Sabbagh $P$, Mohammadnia-Afrouzi M, Javanian M, Babazadeh A Koppolu V, Vasigala VR, et al. Diagnostic methods for helicobacter pylor infection: Ideals, options, and limitations. Eur J Clin Microbiol. Infect Dis 2019;38:55-66. 
42. Abd Rahim MA, Johani FH, Shah SA, Hassan MR, Abdul Manaf MR. $<$ sup $>13<$ /sup $>$ C-urea breath test accuracy for $<$ i $>$ helicobacter pylori $<$ / i $>$ infection in the asian population: A meta-analysis. Ann Glob Health. $2019 ; 85$.

43. Epstein SE. The multiple mechanisms by which infection may contribute to atherosclerosis development and course. Circ Res. 2002;90:2-4.

44. Nam SY, Ryu KH, Park BJ, Park S. Effects of helicobacter pylori infection and its eradication on lipid profiles and cardiovascular diseases. Helicobacter. 2015;20:125-32.

45. Zicha J, Kunes J, Devynck MA. Abnormalities of membrane function and lipid metabolism in hypertension: A review. Am J Hypertens. 1999;12:315-31.
46. Algood HM, Cover TL. Helicobacter pylori persistence: An overview of interactions between h. Pylori and host immune defenses. Clin Microbiol Rev. 2006;19:597-613

47. Rodriguez-Iturbe B, Pons $\mathrm{H}$, Johnson RJ. Role of the immune system in hypertension. Physiol Rev. 2017;97:1127-64.

48. Wang W, Jiang W, Zhu S, Sun X, Li P, Liu K, et al. Assessment of prevalence and risk factors of helicobacter pylori infection in an oilfield community in hebei, china. BMC Gastroenterol. 2019;19:186.

49. Kannel WB, Hjortland MC, MCNamara PM, Gordon T. Menopause and risk of cardiovascular disease: The framingham study. Ann Intern Med. 1976;85:447-52. 